Pis'ma v ZhETF

\title{
Evidence for fine tuning of fermionic modes in lattice gluodynamics
}

\author{
F. V. Gubarev*, S. M. Morozov*, M. I. Polikarpov*, V.I. Zakharov ${ }^{+}$ \\ Institute of Theoretical and Experimental Physics, B. Cheremushkinskaya, 25, Moscow, 117259, Russia
}

Max-Planck Institut für Physik, Föhringer Ring 6, 80805, München, Germany

Submitted 18 July 2005

\begin{abstract}
We consider properties of zero and near-zero fermionic modes in lattice gluodynamics. The modes are known to be sensitive to the topology of the underlying gluonic fields in the quantum vacuum state of the gluodynamics. We find evidence that these modes are fine tuned, that is exhibit sensitivity to both physical (one can say, hadronic) scale and to the ultraviolet cut off. Namely, the density of the states is in physical units while the localization volume of the modes tends to zero in physical units with the lattice spacing tending to zero. We discuss briefly possible theoretical implications and also include some general, review-type remarks.
\end{abstract}

PACS: 11.15.Ha, 11.30.Rd, 12.38.Gc

\section{TWO SCALES OF QCD}

In this note we will consider properties of vacuum 'fluctuations within lattice formulation of Yang Mills theories. For simplicity, we concentrate on the case of pure gluodynamics, with no dynamical fermions. What 'is specific for the lattice formulation (see, e.g., [1) is that it is a field theory in Euclidean space-time. The action reads:

$$
S=\frac{1}{4 g^{2}} \int d^{4} x G_{\mu \nu}^{a}(x) G_{\mu \nu}^{a}(x)
$$

'where $G_{\mu \nu}^{a}(x)$ is the non-Abelian field strength tensor and $a$ is the color index, $\mathrm{g}$ is the coupling. We will 'consider actually the $\mathrm{SU}(2)$ case, $a=1,2,3$. Using action (11) one generates vacuum field configurations, $\left\{A_{\mu}^{a}\right\}$, where $A_{\mu}^{a}$ is the gauge potential and performs further measurements on these fields.

As any renormalizable theory, quantum gluodynamics exhibits two scales, infrared and ultraviolet. Moreover, the ultraviolet cut off is introduced explicitly, through a finite lattice spacing $a$. The infrared scale, $\Lambda_{Q C D}$, on the other hand is emerging dynamically:

$$
\Lambda_{Q C D}^{2} \approx \frac{1}{a^{2}} \cdot \exp \left(-b_{0} / g^{2}(a)\right),
$$

where $b_{0}$ is a constant, $g(a)$ is the bare coupling constant normalized at the lattice spacing, $\Lambda_{Q C D}$ characterizes the scale where the running coupling is of order unit. As $a \rightarrow 0$, the bare coupling squared, $g^{2}(a)$ tends to zero as an inverse $\log$ of $\left(a \cdot \Lambda_{Q C D}\right)$. If one changes the lattice spacing $a$ and modifies $g(a)$ according to the rules of the renormgroup the scale $\Lambda_{Q C D}$ does not depend on $a$.

Lattice simulations allow to study directly vacuum fluctuations and the both scales, $\Lambda_{Q C D}$ and $1 / a$ get manifested in the vacuum fluctuations. In particular, the zero-point fluctuations are sensitive to the ultraviolet cut off. One can measure them, for example, by studying the gluon condensate, or the vacuum expectation value of the gluonic field strength tensor squared:

$$
\left\langle 0\left|\frac{g^{2}}{32 \pi^{2}}\left(G_{\mu \nu}^{a}\right)^{2}\right| 0\right\rangle \approx \frac{\text { const }}{a^{4}}\left\{1+\Sigma_{k} a_{k} g^{2 k}(a)\right\}
$$

where $a_{k}$ are coefficients of the perturbative series. The matrix element (3) is divergent as the fourth power of the ultraviolet cut off. This is the well known divergence of the density of the vacuum energy in field theory, which arises due to the zero-point fluctuations. High gluonic frequencies dominate the matrix element (3) because of the phase space associated with these fluctuations. One can say that zero-point fluctuations represent an example of entropy dominated fluctuations.

Situation looks absolutely different if one considers topological charge. The density of the topological charge is given by:

$$
Q(x)=\frac{g^{2}}{32 \pi^{2}} \epsilon_{\mu \nu \rho \sigma} G_{\mu \nu}^{a}(x) G_{\rho \sigma}^{a}(x),
$$

where $\epsilon_{\mu \nu \rho \sigma}$ is the totally antisymmetric tensor. One can readily show that $Q(x)$ is a full derivative. As a result, all perturbative fluctuations do not contribute to the topological charge. On the other hand, probability to find a non-perturbative fluctuation of size $\rho$ is suppressed for small $\rho$ as $\exp \left(-\right.$ const $\left./ g^{2}(\rho)\right)$ where $g(\rho)$ is the running constant. This factor grows fast with growing $\rho$ and is somehow smoothened out at $\rho \sim \Lambda_{Q C D}^{-1}$ where the running of the coupling cannot be calculated reliably. As a result, topologically nontrivial fluctuations have typical size of order $\Lambda_{Q C D}^{-1}$ and are absolutely 
negligible on the scale of $a$. In particular, instantons are topologically non-trivial and all the factors mentioned above are known explicitly. Instantons represent fluctuations whose probability is determined primarily by action, not entropy (for a review of instantons, in the context of the lattice measurements see, e.g., [2]).

A common viewpoint is that confinement is due to vacuum fluctuations on the scale $\Lambda_{Q C D}$, i.e. is determined by soft, semiclassical fields. The expectations can be confronted with lattice measurements on the fully quantum, vacuum state of gluodynamics. And most recently there has been emerging evidence [3] that the confining fields are actually of a third type, exhibiting both infrared and ultraviolet scales. Both action and entropy are very large but balance each other almost exactly. One can call them fine tuned fluctuations, for a review see 4 .

\section{FINE TUNING}

Imagine that a relativistic system has size $r_{0}$. Then, typical momenta should be of order $p \sim 1 / r_{0}$. Respectively, one expects that typical masses are of order $m^{2} \sim 1 / r_{0}^{2}$. If, on the other hand, observed masses are much smaller than $r_{0}^{-2}$, one calls such a case fine tuning.

The notion of fine tuning has been discussed most thoroughly in connection with the Higgs physics. The problem here is that to fulfill its role as a part of a renormalizable theory of weak interactions the Higgs particle should have mass of order $100 \mathrm{GeV}$, like intermediate bosons of weak interactions. On the other hand, if Higgses are point-like particles down to scale $r_{0}$, then radiative corrections to the Higgs mass are of order

$$
\Delta m_{H}^{2} \sim \alpha r_{0}^{-2}
$$

where $\alpha$ is the electromagnetic coupling. If $r_{0}$ is, say, of order of inverse Planck mass, fine tuning of the radiative correction and of the bare mass is required.

To resolve the puzzle of fine tuning, if it is observed experimentally, one usually invokes hidden symmetry. In the Higgs case, for example, one of the favorite candidates for such a symmetry now is supersymmetry.

In case of Yang-Mills theories, one usually does not expect to confront the problem of fine tuning. However, recently it was found that the field configurations which are responsible for the confinement appear fine tuned. In particular, there is an ample evidence that so called central vortices, for review see, e.g., [5], are responsible for the confinement. The central vortices represent closed two-dimensional surfaces whose total area scales in physical units:

$$
A_{\text {tot }} \approx 24 V_{\text {tot }} \mathrm{fm}^{-2}
$$

where $V_{t o t}$ is the total volume of the lattice. On the other hand, the non-Abelian action associated with the surfaces is ultraviolet divergent [3]:

$$
S_{t o t} \approx 0.55 \frac{A_{t o t}}{a^{2}} .
$$

Combining observations (6) and (7) one concludes that the suppression of the fluctuations due to the action (7) is to be nearly compensated by enhancement due to the entropy. That is, the fluctuations are fine tuned.

The observations above are pure empirical. Theoretically, it is natural to speculate again that there is a hidden symmetry which ensures the observed fine tuning. Moreover, the only symmetry which can come into consideration is the conjectured duality between YangMills theories and string theories [6]. However, such a connection is pure speculative at the moment [4. We mention this possibility just to emphasize that further studies of the fine tuning in Yang-Mills theories are of great interest. In this paper, we address probable manifestation of the fine tuning in fermionic zero modes.

\section{WITTEN-VENEZIANO, BANKS-CASHER RELATIONS}

We will study properties of low-lying modes of the Dirac operator,

$$
D_{\mu} \gamma_{\mu} \psi_{n}=\lambda_{n} \psi_{n}
$$

where $\gamma_{\mu}$ are the Dirac matrices and the covariant derivative $D_{\mu}$ is constructed on the gauge potential $A_{\mu}^{a}$ generated as a vacuum field configuration. The Dirac equation is solved numerically. Moreover, one generates many configurations and studies the properties of the modes with low values of $\lambda_{n}$. Note that both $\lambda_{n}$ and the volume occupied by the $\mathrm{n}$-th wave function $\psi_{n}$ are gauge invariant quantities.

There exists a rich literature on the low-lying Dirac eigenmodes (LDEs). Most commonly, one uses the instanton model of the vacuum, for review and further references see, e.g., [2]. The instantons are localized solutions with non-trivial topological charge and there are exact zero fermionic modes associated with them:

$$
n_{+}-n_{-}=N_{f} \cdot Q_{t o p}
$$

where $n_{ \pm}$are the number of zero modes with positive or negative chirality, $N_{f}$ is the number of quark flavors and the topological charge $Q_{t o p}=1$ for an instanton. 
In the physical vacuum, the instanton solutions are distorted. Indeed, there are neighboring instantons (or anti-instantons) and the instanton fields are modified because of that. The corresponding fermionic zero modes are becoming near-zero modes.

There are some generic features which are predicted to survive the modifications. First, consider exact zero modes, for a given lattice volume $V_{t o t}$. The exact zero modes are related to the total topological charge $Q_{t o p}$. On average, $Q_{t o p}=0$. The value of $Q_{t o p}^{2}$ fluctuates, however. The instanton-like picture presumes that there are lumps of topological charge close to $Q_{t o p}= \pm 1$ occupying sub-volumes of order $\Lambda_{Q C D}^{-4}$. There are no fluctuations of the topological charge on the scale of the lattice spacing $a$. Indeed, instantons are topological excitations with smallest action possible and the probability to find instanton of size of order $a$ is proportional to a high power of $a$. Quantitatively, the strength of the topological fluctuations is related to the $\eta^{\prime}$ mass [7]:

$$
\chi_{t} \equiv \frac{\left\langle Q_{\text {top }}^{2}\right\rangle}{V_{\text {tot }}} \approx \frac{m_{\eta^{\prime}}^{2} f_{\pi}}{2 N_{f}} \approx(213 \mathrm{MeV})^{4},
$$

where the numerical value is borrowed from Ref [15].

There are also near-zero fermionic modes whose total number is proportional to the total volume and which are associated, in the zero approximation, with the original lumps of the topological charge, see above. Since the modes are nearly degenerate, the interaction between instantons results in the delocalization of these zero modes, for details see, e.g., 2]. Quantitatively, this generic feature of the instantonic vacuum is manifested through the Banks-Casher relation [8]:

$$
\langle\bar{\psi} \psi\rangle=-\pi \rho\left(\lambda_{n} \rightarrow 0\right),
$$

where $\rho\left(\lambda_{n} \rightarrow 0\right)$ is the density of the (delocalized) zero modes in the limit of infinite volume $V_{t o t}$. For finite lattice volumes these modes are near-zero.

Thus, Eqs (10) and (11) predict that the number of exact zero modes is proportional to $\sqrt{V_{t o t}}$ while the number of near-zero modes is proportional to the total volume, $V_{\text {tot }}$. Moreover, the instantonic picture fixes the scaling laws according to which the corresponding coefficients of proportionality are in physical units and not dependent on the lattice spacing $a$.

\section{MEASUREMENTS}

We are using the overlap Dirac operator [9]. The advantage of the overlap operator is that it preserves the chiral symmetry and allows to study the properties of the Dirac modes from first principles [10]. There were actually many studies of the Witten-Veneziano and Banks-Casher relations on the lattice. However they used mostly such versions of the lattice fermions that the topological charge has intrinsic ultraviolet noise. To avoid this, one has to modify, smoothen the original gauge fields which fluctuate on the lattice spacing $a$, for review see, e.g., 11. As a result, no dependence on $a$ could actually be measured. The use of the overlap fermions allows to study original gauge fields configuration, see, in particular, [12]. Study of dependence on the lattice spacing $a$ of various observables is one of the main objectives of the present paper.

More explicitly, the massless overlap Dirac operator is given by [9]:

$$
D=\frac{\rho}{a}\left(1+\frac{A}{\sqrt{A A^{\dagger}}}\right), \quad A=D_{W}-\frac{\rho}{a},
$$

where $A$ is the Wilson Dirac operator with negative mass term. Anti-periodic (periodic) boundary conditions in time (space) directions were employed. It turns out that the optimal value of $\rho$ parameter is 1.4. Furthermore, we have used the minmax polynomial approximation 13 to compute the sign function $\operatorname{sign}(A)=A / \sqrt{A A^{\dagger}} \equiv \gamma_{5} \operatorname{sign}(H)$, where $H=\gamma_{5} A$ is hermitian Wilson Dirac operator. In order to improve the accuracy and performance about one hundred lowest eigenmodes of $H$ were projected out. Note that the eigenvalues of (12) lies on the circle of radius $\rho$ centered at $(\rho, 0)$ in the complex plane. In order to relate them with continuous eigenvalues of the Dirac operator the circle was stereographically projected onto the imaginary axis 14.

The calculations were performed on two subsets (Table 2) of statistically independent $S U(2)$ quenched configurations generated with standard Wilson action. For the subset A the gauge coupling was chosen is such a way that the physical volume remains the same, the corresponding lattice spacings were determined by interpolating the data of Ref. 15]. The subset B was used to determine the IPR volume dependence at fixed spacing $a=0.1394(8) \mathrm{fm}$.

\section{RESULTS FOR TOPOLOGICAL SUSCEPTIBILITY}

Let us address first the issue of the scaling of topological susceptibility $\chi_{t}=\left\langle Q_{t o t}^{2}\right\rangle / V_{t o t}$, where the topological charge is defined by the index of overlap Dirac operator $Q_{t o p}=n_{+}-n_{-}$and $n_{ \pm}$is the number of exact zero modes with positive (negative) chirality. The topological susceptibility calculated on the subset A 


\begin{tabular}{|c|c|c|c|c|}
\hline$\beta$ & $a, \mathrm{fm}$ & $L_{s}$ & $L_{t}$ & $V^{p h y s}, \mathrm{fm}$ \\
\hline \multicolumn{5}{|c|}{ Subset A } \\
\hline 2.3493 & $0.1397(15)$ & 10 & 10 & $3.8(2)$ \\
\hline 2.3772 & $0.1284(15)$ & 10 & 14 & $3.8(2)$ \\
\hline 2.3877 & $0.1242(15)$ & 10 & 16 & $3.8(2)$ \\
\hline 2.4071 & $0.1164(15)$ & 12 & 12 & $3.8(2)$ \\
\hline 2.4180 & $0.1120(15)$ & 12 & 14 & $3.8(2)$ \\
\hline 2.4273 & $0.1083(15)$ & 12 & 16 & $3.8(2)$ \\
\hline 2.4500 & $0.0996(22)$ & 14 & 14 & $3.8(3)$ \\
\hline 2.5000 & $0.0854(4)$ & 16 & 18 & $3.92(7)$ \\
\hline \multicolumn{5}{|c|}{ Subset B } \\
\hline 2.3500 & $0.1394(8)$ & 10 & 10 & $3.8(1)$ \\
\hline 2.3500 & $0.1394(8)$ & 10 & 14 & $5.3(1)$ \\
\hline 2.3500 & $0.1394(8)$ & 12 & 12 & $7.8(2)$ \\
\hline 2.3500 & $0.1394(8)$ & 12 & 18 & $11.7(2)$ \\
\hline 2.3500 & $0.1394(8)$ & 14 & 14 & $14.5(3)$ \\
\hline 2.3500 & $0.1394(8)$ & 14 & 16 & $16.5(4)$ \\
\hline \multicolumn{5}{|c|}{ Simulation parameters. } \\
\hline \multicolumn{5}{|l|}{260} \\
\hline \multicolumn{5}{|l|}{250} \\
\hline \multicolumn{5}{|l|}{240} \\
\hline \multicolumn{5}{|l|}{$>^{230}$} \\
\hline$\sum_{\Sigma}^{d} 220$ & \multicolumn{4}{|c|}{$\underset{x}{+} 210$} \\
\hline${ }^{-} \times 0$ & & & & \\
\hline \multicolumn{5}{|l|}{190} \\
\hline \multicolumn{5}{|l|}{180} \\
\hline 170 & 0.005 & $\begin{array}{l}0.0 \\
a^{2}\end{array}$ & 0.015 & 0.02 \\
\hline
\end{tabular}

Scaling of topological susceptibility with lattice spacing; solid curve is the best fit $\chi^{1 / 4}=\chi_{0}^{1 / 4}+c a^{2}$. Square corresponds to the conventional value $(213(3) \mathrm{MeV})^{4}$, 15]

scales up to the $a^{2}$ corrections (Figure 11) and equals to $\chi_{0}=(225(3) \mathrm{MeV})^{4}$ which is in good agreement with the conventional value $(213(3) \mathrm{MeV})^{4}$ [15.

The observed scaling of the topological susceptibility confirms that the discretization errors and the finite volume effects are small. Note that the Wilson gauge action is plagued by lattice dislocations and one might have speculated that spurious fermionic zero modes are generated. However, Figure 1 confirms that the dislocations are inessential for overlap Dirac operator which is insensitive to the ultraviolet noise.

Moreover, we have measured the density of near-zero

\begin{tabular}{l}
$N_{\text {conf }}$ \\
\hline 300 \\
91 \\
198 \\
179 \\
149 \\
198 \\
200 \\
196 \\
\hline
\end{tabular}

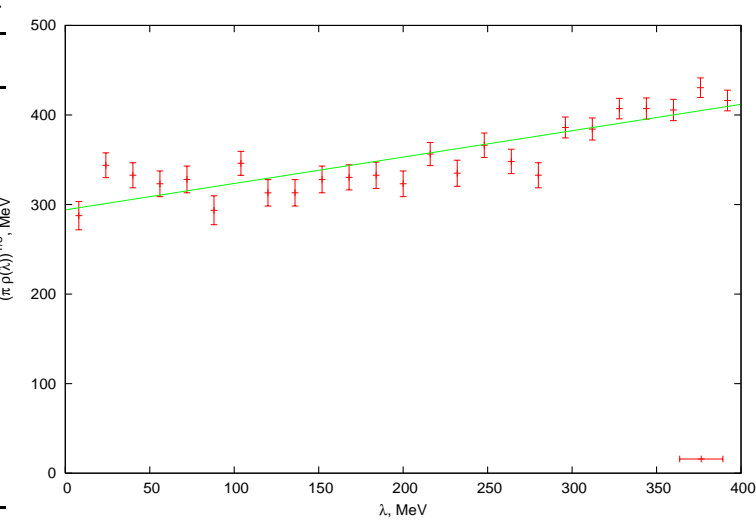

The spectral density of eigenmodes of the overlap Dirac

100 operator and its interpolation to $\lambda_{n} \rightarrow 0$ at $\beta=2.5$

100 on $18 \times 16^{3}$ lattice. The value of the condensate $\langle\bar{\psi} \psi\rangle$, 100 Eq. (11), is perfectly consistent with results of Ref. [16].

100

94

42

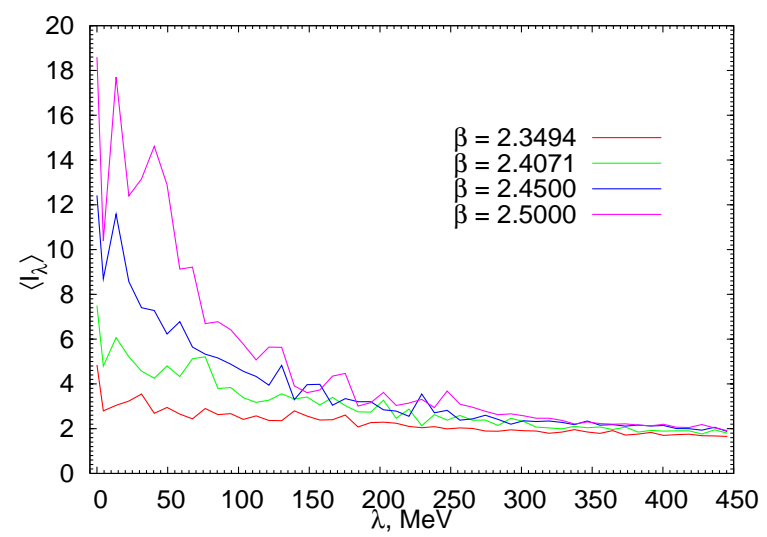

IPRs for LDEs at various lattice spacings and fixed physical volume. The "mobility edge" $\lambda_{c r} \approx 200 \mathrm{MeV}$ is clearly seen.

modes, see Figure 2 The value of the condensate $\langle\bar{\psi} \psi\rangle$ determined through the Banks-Casher relation turns to be close to the value obtained earlier with the Wilson fermions, see, for instance, [16.

\section{SHRINKING OF THE VOLUME OCCUPIED BY FERMIONIC MODES}

A natural measure of the eigenmodes localization is provided by the inverse participation ratio (IPR) $I_{\lambda}$ which is defined as follows (for review and applications see for example Ref. [17]). Let $\rho_{\lambda}(x)$ be the normalized bilinear

$$
\rho_{\lambda}(x)=\psi_{\lambda}^{\dagger}(x) \psi_{\lambda}(x), \quad \sum_{x} \rho_{\lambda}(x)=1,
$$

where $\psi_{\lambda}(x)$ is the eigenmode of the overlap Dirac operator in the given gauge field background with virtuality 
$\lambda, D \psi_{\lambda}=\lambda \psi_{\lambda}$. Then for any finite volume $V$ the IPR $I_{\lambda}$ is defined by

$$
I_{\lambda}=V \sum_{x} \rho_{\lambda}^{2}(x)
$$

and characterizes the inverse fraction of sites contributing to the support of $\rho_{\lambda}(x)$. Note that for delocalized modes $\rho_{\lambda}(x)=1 / V$ and hence $I_{\lambda}=1$, while an extremely localized mode, $\rho_{\lambda}(x)=\delta_{x, x_{0}}$, is characterized by $I_{\lambda}=V$. Moreover, for eigenmodes localized on fraction $f$ of sites (so that $\sup \rho_{\lambda}=V_{f}=f V$ ) we have $I_{\lambda}=V / V_{f}=1 / f$. If we allow for a mixture of both localized and extended modes the average value of IPR is given by:

$$
\left\langle I_{\lambda}\right\rangle=c_{0}+c_{1} V / V_{f} .
$$

Usually [17, one considers localization only in terms of the total volume. Very recently, there appeared data that the localization volume can depend on the lattice spacing as well. Namely, such effect was observed first in Ref. 18 for fermionic modes (in a particular version of lattice fermions, so called Asqtad fermions). Most recently localization properties of scalar probe particles were investigated 19] and, again, strong dependence on $a$ was observed. Observed dependence on the lattice spacing seems specific for dynamics of Yang-Mills theories.

The localization properties of LDEs in our measurements are illustrated on Figure 3 where we plot the inverse participation ratios for modes $0 \leq \lambda \leq 450 \mathrm{MeV}$ at various spacings and fixed physical volume (subset A, Table 2). One can see that there is a critical value, $\lambda_{c r} \approx 200 \mathrm{MeV}$, above which all the states are in fact delocalized and their IPRs are lattice spacing independent. However, for small eigenvalues below $\lambda_{c r}$ the value of IPR grows and one cannot exclude presence of localized states. The mixture of localized and extended modes could be quantitatively characterized by the volume dependence of IPR at fixed lattice spacing, see Eq (14). We have computed the average value $I_{l}=\sum_{\lambda}\left\langle I_{\lambda}\right\rangle / N_{l}$ of IPRs for modes $0<\lambda \leq \Lambda=50 \mathrm{MeV}$, where $N_{l}$ is the total number of modes in this interval, and the analogous quantity $I_{0}$ for exact zero modes. Fitting the data to Eq. (14) we found for exact zero modes $c_{0}=3.7(5), c_{1}=0.38(5)$ with $\chi / n . d . f=3$, and for lowlying modes $c_{0}=1.9(2), c_{1}=2.26(3)$ with $\chi / n . d . f=2$.

Moreover, one can see from Figure 3 that the LDE's localization degree depends non-trivially on the lattice resolution. To quantitatively investigate this dependence we have plotted $I_{0}$ and $I_{l}$ against the lattice spacing on Figure 5 It turns out that our results are highly robust with respect to the actual value of $\Lambda$ as long as $\Lambda \lesssim 50 \mathrm{MeV}$ so that we could take $\Lambda=50 \mathrm{MeV}$ to

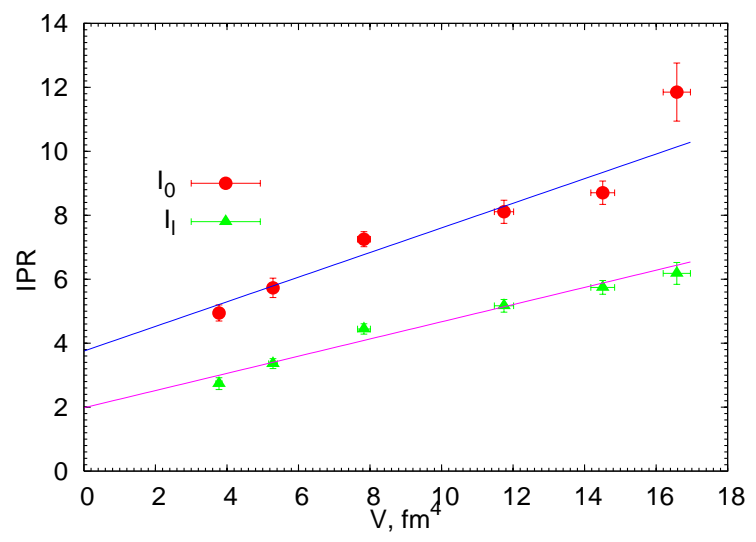

Volume dependence of $I_{0}, I_{l}$ at fixed lattice spacing.

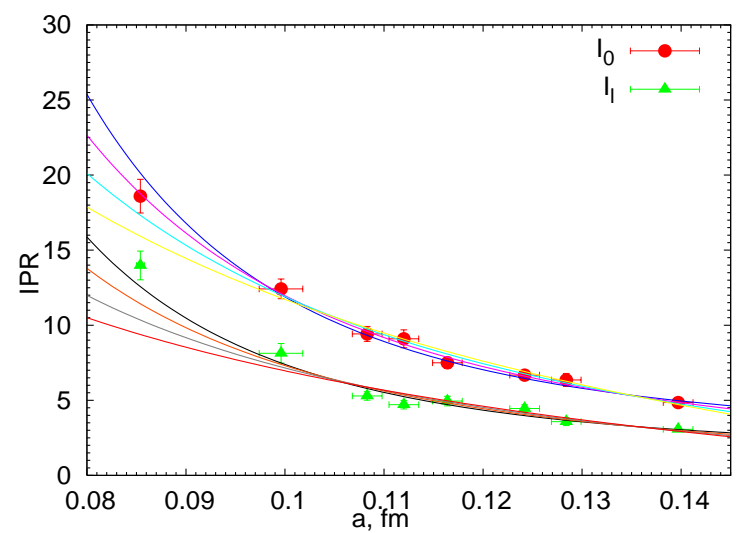

Scaling of $I_{0}$ and $I_{l}$ with $a \rightarrow 0$. Lines correspond to the fitting curves (15) for $d=0,1,2,3$.

improve the statistics. It follows from Figure 5 that the inverse participation ratios for both zero and low lying eigenmodes seem to be divergent in the limit $a \rightarrow 0$. Note that the average values of IPRs are higher than those of Ref. [18.

Furthermore, it is straightforward to estimate the dimensionality $d$ of the objects which localize the low lying eigenmodes. Indeed, at fixed physical volume we have

$$
I_{\lambda}=V / V_{f}=V a^{4} /\left(V_{f} a^{4}\right)=a^{d-4} V^{p h y s} / V_{f}^{p h y s}
$$

and therefore the lattice spacing dependence is given by

$$
\left\langle I_{\lambda}\right\rangle=b_{0}+b_{1} \cdot a^{d-4} .
$$

We fitted our data to Eq. (15) for $d=0,1,2,3$, the fitting curves are shown on Figure 5 As a matter of fact the IPR data for low lying modes strongly suggest that the dimensionality of underlying objects is zero, $d=0$; the relevant $\chi^{2} / n . d . f$. is 2.3 which should be compared with its values $3.4(d=1)$ and $4.9(d=2)$. As far as 
the exact zero modes are concerned our data favor one dimensional $(d=1)$ localization regions; $\chi^{2} / n$.d.f. in this case is 0.38 while it is 0.95 for $d=0$ and 0.85 for $d=2$. The error bars are not small enough, however, to rule out reliably that the exact zero modes in the limit $a \rightarrow 0$ are localized also on point-like objects.

\section{EFFECT OF THE REMOVAL OF THE VORTICES}

Thus, our results indicate fine tuning of the fermionic modes. Namely, their volume shrinks without affecting the eigenvalues. We have already mentioned (see Eqs. (6.77) that fine tuning was observed first for the central vortices. A natural question arises, whether the fine tuning of the fermionic modes is related to existence of the fine tuned vortices. A standard way to probe such a relation is the so called removal of the vortices [20, 21]. Namely one multiplies the original link matrices $U_{\mu}(x)$ by their $Z_{2}$ projected values:

$$
U_{\mu}(x) \rightarrow U_{\mu}(x) \cdot\left(Z_{2}\right)_{\mu}(x) .
$$

One can show that the modification (16) affects (up to a gauge transformation) only a $3 \mathrm{~d}$ fraction of the total $4 \mathrm{~d}$ volume of the lattice [22]. In other words, this fraction is proportional to $\left(a \cdot \Lambda_{Q C D}\right)$ and vanishes in the limit of $a \rightarrow 0$. On the other hand, the vortices are removed. For

The impact of the change (16) on the properties of LDEs is illustrated on Figures [6] 7 Namely, all the low-lying modes, with exception of approximately $4 \%$ of exact zero modes, disappear ${ }^{1)}$. For the modes remaining in the spectrum the value of IPR is of order unit. In other words, removal of the vortices destroys topology-related Dirac modes.

As is mentioned above the change (16) affects a $3 \mathrm{~d}$ sub-volume of the whole lattice. Further information on the manifold crucial for the chirality breaking can be provided by observations on the shrinking of the localization volume as function of the lattice spacing. If indeed, the volume shrinks to points, $\mathrm{d}=0$, as favored by the data above, then it is only a small subspace of the $3 \mathrm{~d}$ volume that is related to chiral symmetry. Pure geometrically, such points could well be the points of self-intersection of the vortices. Theoretically, possible connection between self-intersections of the vortices and zero modes was considered, within a particular model, in Ref. 23. In principle, this conjecture on the relation

1) Note that a similar effect was observed in Ref. 21] where chirally improved lattice Dirac operator was studied.

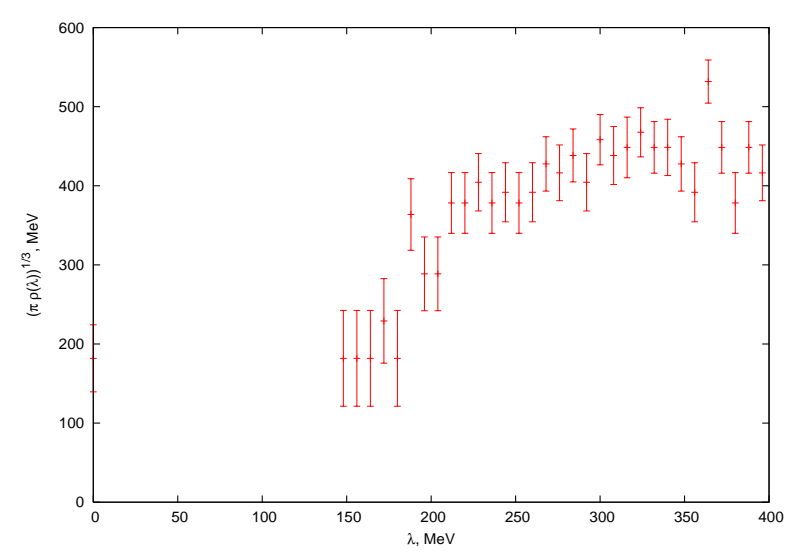

The spectral density of overlap Dirac operator eigenmodes for vortex removed configurations. The lattices used are $18 \times 16^{3}$ at $\beta=2.5$.

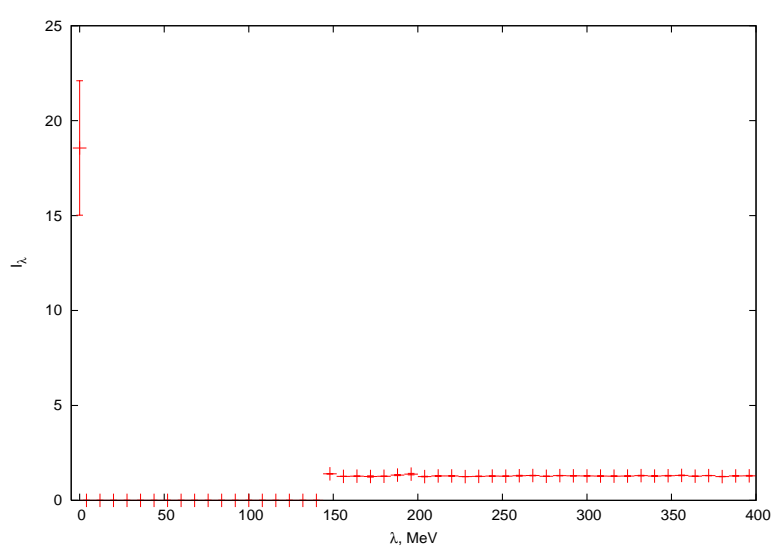

IPR for LDEs for vortex removed configurations obtained at $\beta=2.5$ on $18 \times 16^{3}$ lattices.

between self-intersections of the vortices and localization of (near) zero modes could be investigated through direct observation. At the moment, however, such data are not available.

\section{CONCLUSIONS}

To summarize, we have studied properties of lowlying Dirac modes in the vacuum state of $\mathrm{SU}(2)$ gluodynamics using overlap fermions. We work with the original gauge fields, which fluctuate on the scale of the lattice spacing, without any smoothening. The WittenVeneziano and Banks-Casher relations predict scaling laws for densities for exact and near zero modes, respectively. Namely, these densities should depend only on the physical scale, $\Lambda_{Q C D}$. From the point of view of the lattice measurements, these are strong constraints. The results of the measurements do agree with the theoretical expectations. 
A novel feature uncovered by the lattice simulations is that the low-lying modes are localized on the volumes which shrink as a power of the lattice spacing $a$. For exact zero modes the power is close to three, $V_{\lambda=0} \sim a^{3}$ while for near-zero modes it is rather four, $V_{\lambda \rightarrow 0} \sim a^{4}$, for error bars see Figure 5

Moreover, the removal of the central vortices from the original configurations eliminates all the topological fermionic modes. This observation favors speculations that the lumps of the topological charge are associated with self-intersections of the vortices.

Note that even if this picture is correct, this does not automatically imply that the instantonic picture is wrong. There could be two dual pictures of the same effect, valid for measurements with various resolutions. In terms of soft fields, instanton-like fluctuations could dominate. In terms of fields measured with fine resolution, of order $a$, the fine-tuned surfaces could be responsible for the lumps of the topological charge. It goes without saying that the hypothesis on the crucial role of the surfaces is to be scrutinized much further in the lattice measurements. If existence of the dual pictures is confirmed by measurements, one could speculate that this, empirical duality reflects duality on the fundamental level. The vortices themselves could be identified with dual strings [4, while their self-intersections could be basic element in the mechanism of the chiral symmetry breaking in the dual picture.

One cannot rule out that the lattice-spacing dependence we found will change on finer lattices. In principle, it could happens that the $\sim a^{-4}$ dependence of IPR shown on Figure 5 will eventually flatten in the limit $a \rightarrow 0$ so that the LDEs localization will identify some 4-dimensional objects of finite physical extent. Note however, that the characteristic size of these objects is definitely less than $0.1 \mathrm{fm}$ and likely to be much smaller than that. Although this scenario is not excluded a priori and requires further investigations, it seems artificial to our mind.

The authors are grateful to J. Greensite, S. Olejnik, G. Schierholz, T. Suzuki and to the members of ITEP lattice group for stimulating discussions. The invaluable assistance of G. Schierholz and T. Streuer in the overlap operator implementation is kindly acknowledged. This work was partially supported by the EU Integrated Infrastructure Initiative Hadron Physics (I3HP) grant under contract RII3-CT-2004-506078 and by grants RFBR-05-02-16306a, RFBR-05-02-17642, RFBR-040216079, RFBR-03-02-16941. F.V.G. was partially supported by INTAS YS grant 04-83-3943.
1. M. Creutz, "Quarks, gluons and lattices" (Cambridge Univ. Press, 1983).

2. T. Schafer and E. V. Shuryak, Rev. Mod. Phys. 70, 323 (1998); D. Diakonov, Prog. Part. Nucl. Phys. 51, 173 (2003).

3. V. G. Bornyakov, M. N. Chernodub, F. V. Gubarev, M. I. Polikarpov, T. Suzuki, A. I. Veselov, V. I. Zakharov, Phys. Lett. B 537, 291 (2002); F. V. Gubarev, A. V. Kovalenko, M. I. Polikarpov, S. N. Syritsyn and V. I. Zakharov, Phys. Lett. B 574, 136 (2003); A. V. Kovalenko, M. I. Polikarpov, S. N. Syritsyn and V. I. Zakharov, Phys. Rev. D 71, 054511 (2005); V. G. Bornyakov, P. Y. Boyko, M. I. Polikarpov and V. I. Zakharov, Nucl. Phys. B 672, 222 (2003); B. L. G. Bakker, A. I. Veselov and M. A. Zubkov, Phys. Lett. B 544, 374 (2002).

4. V.I. Zakharov, AIP Conf. Proc. 756, 182 (2005); hep-ph/0312210

5. J. Greensite, Prog. Part. Nucl. Phys. 51, 1 (2003).

6. J.M. Maldacena, Adv. Theor. Math. Phys.,2, 231 (1998); A.M. Polyakov, Int. J. Mod. Phys. A14, 645 (1999).

7. E. Witten, Nucl. Phys. B156, 269 (1979); G. Veneziano, Nucl. Phys. B159, 213 (1979).

8. T. Banks, A. Casher, Nucl. Phys. B169, 103 (1980).

9. H. Neuberger, Phys. Lett. B 417, 141 (1998); H. Neuberger, Phys. Lett. B 427, 353 (1998).

10. M. Luscher, Phys. Lett. B 428, 342 (1998).

11. M. Teper,Nucl. Phys. Proc. Suppl. 83, 146 (2000).

12. I. Horvath, S.J. Dong, T. Drape, N. Isgur , F.X. Lee, K.F. Liu, J. McCune, H.B. Thacker, J.B. Zhang, Phys. Rev. D66, 034501 (2002); I. Horvath et al., Phys. Rev. D 68, 114505 (2003); I. Horvath, S. J. Dong, T. Draper, F. X. Lee, K. F. Liu, H. B. Thacker and J. B. Zhang, Phys. Rev. D 67, 011501 (2003).

13. L. Giusti, C. Hoelbling, M. Luscher and H. Wittig, Comput. Phys. Commun. 153, 31 (2003).

14. S. Capitani, M. Gockeler, R. Horsley, P. E. L. Rakow and G. Schierholz, Phys. Lett. B 468, 150 (1999).

15. B. Lucini and M. Teper, JHEP 0106, 050 (2001).

16. S. J. Hands and M. Teper, Nucl. Phys. B347, 819 (1990).

17. B. Kramer, A. MacKinnon, Rep.Prog.Phys. 56, 1469 (1993); C. Gattringer et al. Nucl. Phys. B617, 101 (2001); T. Kovacs, Phys. Rev. D67, 094501 (2003).

18. C. Aubin et al. [MILC Collaboration], hep-lat/0410024

19. J. Greensite, S. Olejnik, M. Polikarpov, S. Syritsyn and V. Zakharov, Phys. Rev. D 71, 114507 (2005).

20. P. de Forcrand and M. D'Elia, Phys. Rev. Lett. 82, 4582 (1999).

21. J. Gattnar et al., Nucl.Phys. B716, 105 (2005).

22. A. V. Kovalenko, M. I. Polikarpov, S. N. Syritsyn and V. I. Zakharov, Phys. Lett. B613, 52 (2005).

23. M. Engelhardt, H. Reinhardt, Nucl. Phys. B567, 249 (2000). 